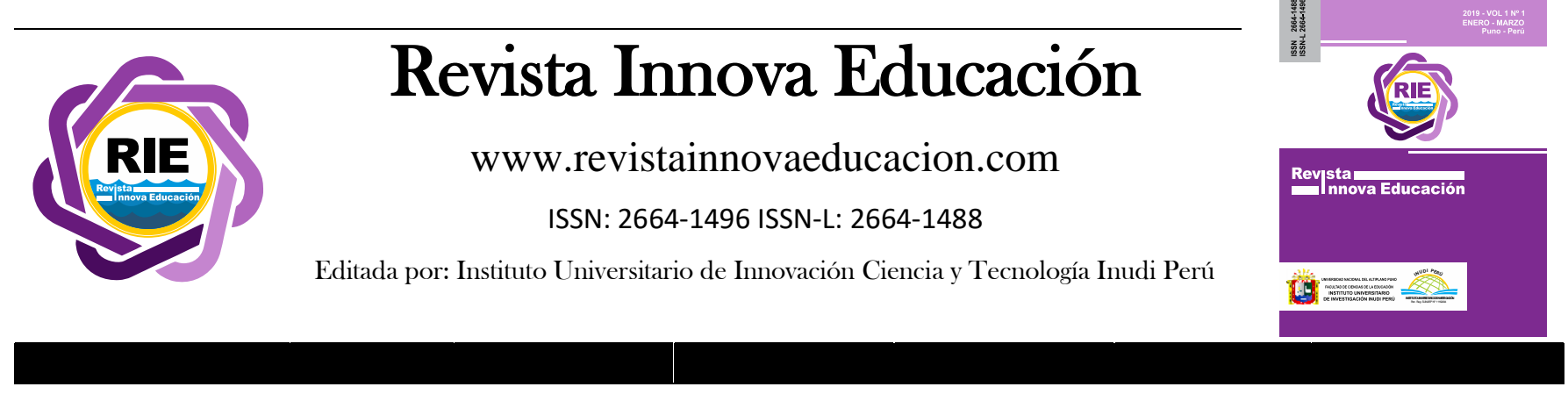

\title{
Bases epistemológicas que sustentan la teoría de las inteligencias múltiples de Howard Gardner en la pedagogía
}

\section{Epistemological foundations underpinning Howard Gardner's theory of multiple intelligences in pedagogy}

\author{
Eber Chura iD; Pedro Huayanca ${ }^{D}$; Martha Maquera \\ DOI: https://doi.org/10.35622/j.rie.2019.04.012
}

Universidad Nacional del Altiplano, Perú

Facultad de Ciencias de la Educación

Recibido el 23/03/2019/ Aceptado el 28/10/2019

\begin{tabular}{|c|c|}
\hline $\begin{array}{l}\text { ARTÍCULO ORIGINAL } \\
\text { PALABRAS CLAVE } \\
\text { Educación, Howard } \\
\text { Gardner, inteligencias } \\
\text { múltiples, pedagogía. }\end{array}$ & $\begin{array}{l}\text { La Teoría de las Inteligencias Múltiples fue propuesta por el estadounidense Howard } \\
\text { Gardner como contrapeso al paradigma tradicional de una inteligencia única y ha } \\
\text { revolucionado la concepción de la inteligencia en la pedagogía. El objetivo fue } \\
\text { determinar las bases epistemológicas de la teoría de las inteligencias múltiples de } \\
\text { Howard Gardner en la pedagogía. El estudio corresponde al enfoque cualitativo de tipo } \\
\text { teórico de análisis retrospectivo para el cual se ha usado la hermenéutica y como técnica } \\
\text { el análisis del contenido. Los resultados y discusión de la investigación subsumen una } \\
\text { interpretación teórica sistemática de autores respecto a las bases epistemológicas que } \\
\text { sustentan la teoría de las inteligencias múltiples en la pedagogía. Se concluye que las } \\
\text { bases epistemológicas de la Teoría de las Inteligencias Múltiples de Howard Gardner } \\
\text { se encuentran en la psicología humanista de la educación, la neuropsicología y el } \\
\text { modelo holístico de la educación. }\end{array}$ \\
\hline
\end{tabular}

\section{KEYWORDS}

Education, Howard Gardner, multiple intelligences, pedagogy.

\begin{abstract}
The Theory of Multiple Intelligences was proposed by the American Howard Gardner as a counterweight to the traditional paradigm of a single intelligence and has revolutionized the conception of intelligence in pedagogy. The objective was to determine the epistemological basis of Howard Gardner's theory of multiple intelligences in pedagogy. The study corresponds to the qualitative approach of the theoretical type of retrospective analysis for which hermeneutics has been used and as a technique the content analysis. The results and discussion of the research subsume a systematic theoretical interpretation of authors regarding the epistemological bases that support the theory of multiple intelligences in pedagogy. It is concluded that the epistemological bases of Howard Gardner's Theory of Multiple Intelligences are found in the humanistic psychology of education, neuropsychology and the holistic model of education.
\end{abstract}




\section{INTRODUCCIÓN}

La inteligencia es el proceso psicológico cognitivo que consiste en usar capacidades y habilidades para solucionar problemas nuevos (Contreras \& Sotomayor, 2013); procede del latín (inter-entre y eligere-elegir) y se puede definir como la capacidad del cerebro de elegir la mejor opción para resolver problemas y dificultades (Castro, Fonseca, \& Reyes, 2014); permitiendo la adaptación eficiente al ambiente físico y social, así como la capacidad para planear, pensar de manera abstracta, comprender ideas complejas, aprender de la experiencia (Ardila, 2011); sin embargo, la evolución de la sociedad y la ampliación del campo del conocimiento y los saberes hicieron que se fuera perfilando un concepto de inteligencia más específico y concreto (Huerta, Giles, \& Rojas, 2010).

La inteligencia es tan importante como controvertido en la historia de la psicología, de ahí que la tarea de esclarecer qué es la inteligencia no resulte fácil sino sólo la dirección de un esfuerzo que debemos realizar colectivamente (González, 2003); podemos organizar los conceptos en psicológicos, biológicos y operativos, tomando el cariz particular de cada una de estas disciplinas (Suárez, Maiz, \& Meza, 2010); sin embargo, las características que asociamos con el concepto de capacidad de solucionar problemas, de razonar, de adaptarse al ambiente, han sido altamente valoradas a lo largo de la historia (Ardila, 2011); en consecuencia la inteligencia es una actividad psíquica compleja que comprende la organización de nuestras habilidades para enfrentar situaciones problemáticas empleando nuevos recursos o procedimientos, resolviendo dichas situaciones de manera eficiente (Chura, 2016).

Existe una gran variedad de teorías explícitas sobre la inteligencia, las mimas que se inscriben en psicométricas, biológicas, del desarrollo y las inteligencia inteligencias múltiples (Villamizar \& Donoso, 2013), aunque históricamente se ha concebido la existencia de una inteligencia única, la cual era susceptible de cuantificación al ser evaluada numéricamente (Macías, 2002); sin embargo, Howard Gardner considera que no existe una única inteligencia, sino que cada persona posee al menos ocho inteligencias: inteligencia lingüística, lógico-matemática, cinestésico-corporal, musical, espacial, naturalista, interpersonal e intrapersonal (Carrillo \& López, 2014); las mismas que se refieren a la capacidad humana de aprender y aplicar ese conocimiento de múltiples maneras (Castro et al., 2014); la diferencia radica en la manera en que se recurre a ellos y se les combina para llevar a cabo diferentes labores, para solucionar problemas y progresar en distintos ámbitos (Sánchez, 2015). La importancia de las inteligencias múltiples radica en que estas amplían el campo de lo definido como "inteligencia" dado que existen personas con diversas capacidades y habilidades (Stecconi, 2006); cada inteligencia tiene un conjunto de operaciones núcleo que sirven para impulsar las diferentes actividades naturales de esa inteligencia (Suárez et al., 2010).

La educación es el mejor medio para desarrollar la inteligencia mediante la tecnología, ciencia, valoración de la cultura, toma de conciencia de las capacidades personales y el desarrollo de la creatividad (Castro et al., 2014), es por ello que los docentes saben intuitivamente que sus alumnos poseen talentos, habilidades, experiencias e intereses variados (Shannon, 2013); en consecuencia, la teoría de las inteligencias múltiples ofrece una sólida fundación para reconocer los diferentes talentos y habilidades de los estudiantes (Emst-Slavit, 2001). La aplicación de la teoría de las inteligencias múltiples al campo de la enseñanza supone una síntesis y una continuación de una serie de enfoques humanistas (S. Gallegos, 2009); de ahí que en el proceso educacional consideramos un área óptima en que se pueden observar las inteligencias múltiples en acción (Gardner, 1983); después de adoptar esta perspectiva más amplia y pragmática, el concepto de "inteligencia" se convierte en un concepto funcional en la psicología educativa (Armstrong, 2017).

La psicología educativa es una ciencia interdisciplinaria que se identifica con dos campos de estudios diferentes, pero interdependientes entre sí: las ciencias psicológicas y las ciencias de la educación 
(Cuevas, 2011), en el primer campo los psicólogos educativos hacen investigación sobre el aprendizaje y la enseñanza trabajando para mejorar la práctica educativa (Pintrich, 2000); y en el segundo campo, la pedagogía es un conjunto de saberes que se aplican a la educación como fenómeno típicamente social y específicamente humano (Romero, 2009); bajo esta perspectiva, se subsume en la praxis educativa las ocho inteligencias que Gardner ha reconocido en todos los seres humanos: la lingüística-verbal, la musical, la lógico-matemática, la espacial, la corporalcinestésica, la intrapersonal, la interpersonal y la naturalista (Blanes, 2016); planteando de este modo una escuela centrada en el individuo, comprometida con el entendimiento óptimo y el desarrollo del perfil cognitivo de cada estudiante (Suárez et al., 2010).

El concepto de educación y humanismo responden a un mismo ideal y estructura, cualquier proceso educativo formal o informal tiene la meta de construir en cada ser humano su humanidad (Cordero, 2007), es así que el paradigma humanista postula a la persona como eje central del modelo y dentro de la perspectiva educativa recomienda el estudio del ser humano de una manera integral (Aizpuru, 2008); en efecto, los alumnos disfrutarían más la escuela si las disciplinas fueran presentadas en diferentes modalidades y el aprendizaje fuera susceptible de evaluación mediante diversas maneras (Sánchez, 2015); bajo esta perspectiva, la neuropsicología se inscribe en el ámbito de la neurociencia cuyo objetivo es unificar el conocimiento de los procesos neurobiológicos y psicobiológicos (Portellano, 2005), y la idea de fondo del holismo, es la de la comprensión de los fenómenos desde la multidimensionalidad, pues la realidad está compuesta de una diversidad de variables que interactúan entre sí (Gluyas, Esparza, \& Romero, 2015).

En consecuencia, las bases epistemológicas de la teoría de las inteligencias múltiples de Howard Gardner se encuentran en la psicología humanista de la educación, así como en la neuropsicología y el modelo holístico de la educación. Gardner ha preparado el escenario para la introducción de las inteligencias múltiples en la psicología educativa y la pedagogía para romper con la concepción cognitiva tradicional de la inteligencia.

\section{MÉTODO}

La investigación corresponde al enfoque cualitativo de tipo teórico hermenéutico. Para la obtención de resultados se aplicó como técnica el análisis de contenido. Todo ello permitió analizar e interpretar los conocimientos planteados acerca de las inteligencias múltiples en la pedagogía para determinar las bases que la originan y fundamentan.

\subsection{Ejes de análisis}

Tabla 1 Corpus de estudio y ejes temáticos

\begin{tabular}{lllll}
\hline Universo de estudio & Ejes de estudio & Unidades de estudio & \\
\hline & & $\begin{array}{l}\text { Concepción psicológica de la } \\
\text { inteligencia. }\end{array}$ & \\
Teoría de las inteligencias & Inteligencia y pedagogía & \\
múltiples de Howard & Concepción de Howard Gardner \\
Gardner en la pedagogía. & $\begin{array}{l}\text { acerca de la inteligencia. } \\
\text { Pedagogía y las inteligencias } \\
\text { múltiples. }\end{array}$ \\
\hline
\end{tabular}




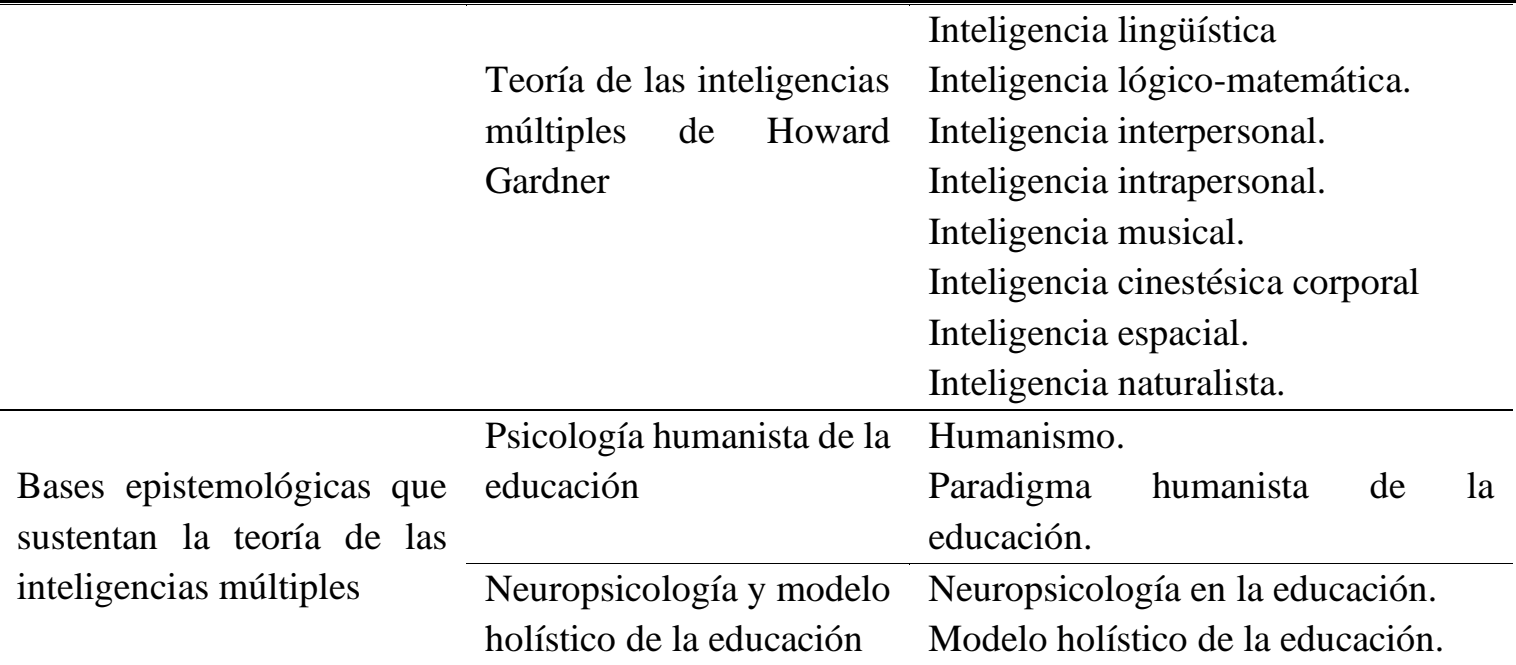

Fuente: Elaboración del autor

\subsection{Procedimientos que se realizaron para el estudio}

En principio se realizó la búsqueda de la literatura en las principales bases de dato de investigaciones científicas: Scielo, Scopus, Doaj, Open thesis, ScienceDirect, Web of Science, Eric, Dialnet, Redalyc y Scholar Google. La información recabada permitió construir el universo de estudio y los ejes de análisis.

\section{RESULTADOS Y DISCUSIÓN}

\subsection{Teoría de las inteligencias múltiples de Howard Gardner en la pedagogía}

La teoría de las inteligencias múltiples que de un tiempo a esta parte se han subsumido en diferentes programas educativos fueron desarrollados por Howard Gardner y constituyen uno de los pilares de la psicología educativa que, encargada de abordar los problemas que presenta la pedagogía, para diseñar métodos y establecer técnicas psicológicas que permitan lograr óptimos niveles de aprendizajes y desarrollo de las diversas capacidades de los estudiantes.

\subsubsection{Inteligencia y pedagogía}

Las concepciones acerca de la inteligencia y la pedagogía han evolucionado conjuntamente con el desarrollo histórico de la psicología como ciencia, muchos estudiosos de la psicología han propuesto sus propios conceptos y estos convergen en lo que llamaremos la concepción psicológica de la inteligencia, sin embrago Howard Gardner ha revolucionado los conceptos tradicionales de la inteligencia con su teoría de las inteligencias múltiples.

Concepción psicológica de la inteligencia. La inteligencia es el proceso psicológico cognitivo superior que consiste en usar capacidades y habilidades del sujeto para solucionar problemas nuevos de manera eficiente. Esta definición quiere decir que, la inteligencia es una facultad del ser humano que le permite adaptarse a diferentes entornos y circunstancias, donde el sujeto usará sus cualidades psicológicas aprendidas para resolver con éxito distintos problemas o determinadas actividades. Al respecto, Contreras \& Sotomayor (2013) definen la inteligencia como el uso de las habilidades y capacidades en la resolución de problemas nuevos que le permiten a un sujeto adaptarse a su medio ambiente. Por otro lado, Morris \& Maisto (2005) sostiene que la inteligencia es una actividad psíquica que influye en nuestro éxito en la escuela, el tipo de trabajo que realizamos, los tipos de recreación e incluso en la elección de los amigos. Además, Rosas (1996) señala que la inteligencia es la capacidad que tiene el hombre para razonar, entender y juzgar debidamente, 
de modo que pueda adaptarse o enfrentarse al mundo que lo rodea; además, la inteligencia se deduce siempre de un hecho realizado. Por su parte, Papalia (2009) sostiene que la inteligencia es el resultado de la interacción entre las capacidades heredadas y las experiencias ambientales para aplicar y utilizar todo ello con el propósito concreto de resolver los problemas de la vida cotidiana. Las posturas de los autores citados confirman nuestra concepción respecto a la inteligencia con lo que consolidamos el resultado de nuestra investigación.

Concepción de Howard Gardner acerca de la inteligencia. Gardner al desarrollar su teoría sostiene que el ser humano no puede tener una sola inteligencia, por lo que ha preparado un escenario para una introducción de las inteligencias múltiples en la pedagogía cognitiva tradicional. El autor es uno de los personajes cuyas aportaciones han tenido más trascendencia en la psicología educativas, desde su concepción la inteligencia es entendida como las distintas facultades intelectuales o competencias que desarrollamos los seres humanos en transcurso de nuestro ciclo vital. Gardner (1983) sostiene que sus estudios de la inteligencia y la cognición indican la existencia de muchas y distintas facultades intelectuales, o competencias, cada una de las cuales puede tener su propia historia de desarrollo. Por su parte, Armstrong (2017) plantea que Gardner aportó un método para trazar la amplia gama de capacidades que posee el ser humano agrupándolas en ocho categorías o "inteligencias" en la pedagogía. Al respecto Maquera (2017) sostiene que las inteligencias múltiples tienen un alto grado de relación con el rendimiento académico. La inteligencia percibida desde la concepción de Howard Gardner no solo es aplicada al campo de la educación y la pedagogía, por el contrario, forma parte la vida cotidiana de los seres humanos. Lo mencionado anteriormente se puede evidenciar en la tesis doctoral de Maldonado (2014), quien investigó la influencia de las inteligencias múltiples en las habilidades emprendedoras: estudio de casos de éxito en Extremadura. Finalmente, Macías (2002) plantea que Gardner rompe con el esquema tradicional de inteligencia dándole al concepto un nuevo significado al referirse con él a una amplia variedad de capacidades humanas, congruente con esto, casi todos podemos reconocer la existencia de la creatividad. Los autores mencionados confirman nuestra postura epistemológica respecto a la teoría de las inteligencias múltiples de Howard Gardner.

Pedagogía y las inteligencias múltiples. La psicopedagogía o psicología educacional es una rama de la psicología aplicada encargada de abordar los problemas de enseñanza y aprendizaje, para diseñar métodos y establecer técnicas psicológicas que permitan lograr óptimos niveles de aprendizajes; del mismo modo aborda temas afines al proceso de enseñanza-aprendizaje tales como orientación vocacional y perfil profesional de las personas. Para Cuevas (2011) la psicología educativa es una ciencia interdisciplinaria que se identifica con dos campos de estudios diferentes, pero interdependientes entre sí: las ciencias psicológicas y las ciencias de la educación. Por su parte, Pintrich (2000) afirma que se acepta actualmente que la psicología educativa es una disciplina que investiga sobre el aprendizaje y la enseñanza, al mismo tiempo, trabaja para mejorar la práctica educativa. Por otro lado, Woolfolk (2010) sostiene que la psicología educativa es la disciplina que estudia los procesos de enseñanza y aprendizaje; aplica los métodos y las teorías de la psicología, aunque también posee los propios. Por su parte, Carrillo \& López (2014) plantean que asumir la teoría de Gardner supone un cambio significativo en el modelo educativo, ya que implica la toma en consideración de las potencialidades innatas del alumnado, de manera que la enseñanza se convierta en un proceso de estimulación de cada una de las inteligencias de la persona. Las citas mencionadas en el párrafo anterior corroboran nuestra propuesta respecto a la pedagogía y las inteligencias múltiples, por lo que se confirma la interdependencia de los conceptos mencionados en el campo de la educación. 


\subsubsection{Teoría de las inteligencias múltiples de Howard Gardner}

De acuerdo a los planteamientos de distintos autores, Gardner propuso su teoría, considerando que la inteligencia no es una sola unidad, sino un conjunto de inteligencias múltiples, cada una de las cuales es un sistema con existencia propia con oposición a los aspectos meramente separados de un sistema mayor: "la inteligencia" y cada inteligencia es independiente de las otras, sin embargo, estas interactúan entre sí. Contreras \& Sotomayor (2013) afirman que, según la teoría de Howard Gardner poseemos ocho inteligencias múltiples relativamente independientes entre sí, conforme a esta teoría distintas clases de inteligencia pueden producir diferentes respuestas, pero válidas a una misma pregunta. Por otro lado, Blanes (2016) sostiene que, hasta el momento, existen ocho inteligencias que Gardner ha reconocido en todos los seres humanos: la lingüística-verbal, la musical, la lógica-matemática, la espacial, la corporalcinestésica, la intrapersonal, la interpersonal, y la naturalista. Siguiendo estos conceptos está implícitamente entendido que las inteligencias múltiples constituyen la base pedagógica para elección de una profesión. Según Llacsa (2011), existe una relación alta entre las inteligencias múltiples y elección vocacional, este afirmación está sustentado en la correlación Pearson entre las variables estudiadas.

Seguidamente identificaremos y desarrollaremos puntalmente ocho inteligencias múltiples de Howard Gardner. La inteligencia lingüística es la capacidad implicada en la producción de textos y comprensión del lenguaje y expresión verbal; la inteligencia lógico-matemática es la capacidad aplicada en la solución de problemas relacionados al cálculo numérico y las operaciones formales; la inteligencia interpersonal es la capacidad para interactuar con las personas; captar sus estados de ánimo, temperamentos, motivaciones e intenciones; la inteligencia intrapersonal es la capacidad para el autoconocimiento de nuestros aspectos internos, acceso a la propia vida emocional, así como a sus sentimientos más profundos; la inteligencia musical es aquella capacidades y habilidad que permiten mejor percepción y producción para las tareas musicales; la inteligencia cinestésica corporal es la capacidad en el control del movimiento corporal, que permite solucionar problemas cinestésico-corporales; la inteligencia espacial es la capacidad que implica configuraciones espaciales de orientación; la inteligencia naturalista es la capacidad para resolver problemas de la naturaleza y trabajar directamente con elementos del medio ambiente.

\subsection{Bases epistemológicas que sustentan la teoría de las inteligencias múltiples}

Las bases científicas o epistemológicas de la Teoría de las Inteligencia Múltiples de Howard Gardner se encuentran en la psicología humanista de la educación, la neuropsicología y el modelo holístico de la educación.

\subsubsection{La psicología humanista de la educación}

La psicología humanista de la educación es una postura epistemológica que se caracteriza por darle primordial importancia al ser humano como un todo, resaltando todas sus cualidades, descubrir la verdadera naturaleza humana y darle sentido racional a la vida; su base filosófica se encuentra en el humanismo existencial.

Humanismo. En la psicología humanista de la educación el ser humano es valorado integralmente con todas sus potencialidades intelectuales, lo que significa que respecto a la inteligencia no solo debemos limitarnos al aprendizaje cognitivo en la pedagogía, por el contrario, entendiendo que el ser humano es una totalidad biopsicosocial debemos desarrollar otros tipos de aprendizajes como el social, afectivo, actitudinal y motor; y para desarrollar mejor estos tipos de aprendizajes recurrimos al desarrollo de las inteligencias múltiples. Velasco (2009) señala que es común asociar la idea de humanismo al saber propio de las disciplinas 
humanísticas que se consolidan como campo del saber, a distintas disciplinas formada por la gramática, la dialéctica y la retórica y abarcan la filosofía, la filología, la historia, la literatura, el teatro, entre otras. Por su parte, Cordero (2007) indica que el concepto de Educación y Humanismo responden a un mismo ideal y estructura; a saber, cualquier proceso educativo, formal o informal, tiene la meta de construir en cada ser humano su humanidad a lo largo de las diferentes etapas de la vida. Por otro lado, Cordura (2013) sostiene que las humanidades son una creación del humanismo, pero "humanismos" hubo varios, antes del que nos interesa aquí, algunos ya en la edad media y otros después de ella, pero todos enfocados en la integridad de ser humano como tal. Estos planteamientos sirven de base epistemológica que sostienen la teoría de las inteligencias múltiples de Howard Gardner en la pedagogía y confirman que el humanismo valora la totalidad de las inteligencias que pueda tener el ser humano.

Paradigma humanista de la educación. En el paradigma humanista de la educación la persona se convierte en el eje fundamental de la educación, esta debe ser concebida de manera integral rescatando todas sus cualidades humanas, mas no así fragmentar su estudio centrándonos en solo en determinadas capacidades de los seres humanos. Respecto a lo mencionado, Aizpuru (2008) afirma que el paradigma humanista postula a la persona como eje central del modelo, dentro de la perspectiva educativa recomienda el estudio del ser humano de una manera integral, como una totalidad. Por otro lado, Cordura (2013) sostiene que lo interesante de la amplia y duradera influencia de la inspiración humanística reside en que, aun allí donde algunos han abandonado las ideas de la libertad y la universalidad del hombre o no han consentido nunca en adoptarlas. Por su parte, Sánchez (2015) considera que los alumnos disfrutarían más la escuela si las disciplinas fueran presentadas en diferentes modalidades y el aprendizaje fuera susceptible de evaluación mediante diversas maneras, así se ampliaría el concepto de inteligencia. Suárez et al., (2010) plantean que la teoría de las inteligencias múltiples se considera de gran importancia para potenciar los aprendizajes de los niños y jóvenes; minimiza los problemas de conducta; incrementa la autoestima en los niños y jóvenes; desarrolla las habilidades de cooperación y liderazgo, y aumenta el interés y la dedicación al aprendizaje. Los autores citados confirman nuestra investigación científica, en consecuencia, el paradigma humanista sirve de base epistemológica para introducir la Teoría de la Inteligencias Múltiples de Howard Gardner en la pedagogía.

\subsubsection{La neuropsicología y el modelo holístico de la educación}

La neuropsicología y el modelo holístico de la educación constituyen las bases epistemológicas de la teoría de las inteligencias múltiples de Howard Gardner en la pedagogía, esta postura científica de la educación se sostiene en distintas investigaciones de la psicología, pedagogía, neurología y filosofía. El modelo holístico de la educación es un fenómeno psicosocial enraizado en las diversas disciplinas humanas y orientada formación integral del ser humano y la neuropsicología el fundamento biológico de la actividad cognitiva.

Neuropsicología en la educación. La neuropsicología o psicofisiología es una rama de la psicología pura normal que estudia el fundamento biológico de la actividad psicológica, así como las bases neurológicas de la conciencia y de la personalidad, para lo cual investiga fundamentalmente el sistema nervioso en relación a los procesos mentales como la inteligencia, el pensamiento, la memoria, etc. Al respecto, Benedet (2012) sostiene que la neuropsicología es el estudio de las relaciones entre la conducta y el cerebro; pero a tenor de la evolución de los conocimientos que han ido acumulando las otras disciplinas a las que está estrechamente vinculada y que hoy se agrupan bajo la denominación de neurociencia. Por otro lado, Fernández, Paúl, \& Maestú (2003) plantean que en las últimas tres décadas la neuropsicología, ha ido cambiando paulatinamente hasta formar lo que hoy se conoce por neuropsicología cognitiva. Por su parte, Portellano (2005) fundamenta que la neuropsicología se inscribe en el ámbito de la neurociencia, que es un abordaje multidisciplinar del estudio del sistema nervioso cuyo objetivo es unificar el conocimiento de los 
procesos neurobiológicos y psicobiológicos. Según Armstrong (2017), Gardner trató con individuos que habían sufrido accidentes o enfermedades que les habían afectado zonas específicas del cerebro. Para Tirapu (2011), en varios casos, las lesiones cerebrales parecían haber perjudicado a una inteligencia concreta, mientras las demás permanecían intactas. Con los fundamentos expuestos por los autores mencionados nuevamente confirmamos nuestra postura científica respecto de la neuropsicología en la educación como base epistemológica de la teoría de las inteligencias múltiples de Howard Gardner en la pedagogía.

Modelo holístico de la educación. El modelo holístico en la educación considera al ser humano como un todo integrado que tiene muchas facultades y potencialidades, por ende, la educación según este modelo debe ser integral y no solamente cognitivo, es decir, debe subsumir en sus programas el desarrollo de las inteligencias múltiples como la musical, cinestésica-corporal, espacial, interpersonal, intrapersonal, naturalista; además de la inteligencia lingüística y lógico-matemática. Barrera Barrera (1999) afirma que la holística se refiere a la manera de ver las cosas enteras, en su totalidad, en su conjunto, en su complejidad, pues de esta forma se pueden apreciar interacciones, particularidades y procesos. Por otro lado, Gluyas et al., (2015) sostienen que la idea de fondo del holismo, es la de la comprensión de los fenómenos desde la multidimensionalidad, pues la realidad está compuesta de una diversidad de variables que interactúan entre sí. Por su parte, Suárez et al., (2010) plantean que, el docente asuma que la inteligencia puede ser potenciada, que podemos hacer niños más inteligentes, trabajando no las limitaciones sino las potencialidades, implica entonces enfatizar en el desarrollo de las habilidades cognitivas. Al respecto, Gallegos (1999) sostiene que, en la educación holista, aprender es un proceso que implica muchos niveles de la conciencia humana como el afectivo, físico, social y espiritual, rebasando por completo lo puramente cognitivo y memorístico. Gluyas et al., (2015) sostienen que el modelo de Educación Holística procura su cabal y pleno desarrollo de plenitud existencial, a través de una educación de naturaleza holística, es decir, integral y multidimensional. Estos fundamentos sustentan que el modelo holístico tiene una aplicación epistemológica en la educación, en consecuencia, los autores citados confirman nuestra teoría respecto a este modelo como base epistemológica de la teoría de las inteligencias múltiples de Gardner.

\section{CONCLUSIONES}

Las bases científicas o epistemológicas de la Teoría de las Inteligencia Múltiples de Howard Gardner, teoría que ha revolucionado la psicología educativa, se encuentran en la psicología humanista de la educación, la neuropsicología y el modelo holístico de la educación. Las inteligencias múltiples propuestas por Howard Gardner, las mismas que están subsumidos en la pedagogía son: lingüística, lógico-matemática, interpersonal, intrapersonal, cinestésica-corporal, musical, espacial y naturalista; las mismas que indistintamente forman parte de la praxis pedagógica.

\section{REFERENCIAS BIBLIOGRÁFICAS}

Aizpuru, M. G. (2008). La Persona como Eje Fundamental del Paradigma Humanista. Acta Universitaria de la Universidad de Guanajuato México, 18(1), 33-40.

Ardila, R. (2011). Inteligencia. ¿qué sabemos y qué nos falta por investigar? Revista Académica Colombiana de Ciencias Exactas, Físicas y Naturales, 35(134), 97-103.

Armstrong, T. (2017). Inteligencias múltiples en el aula (1ra Ed.). Barcelona: Paidós Educación.

Barrera, M. F. (1999). Holística, comunicación y cosmovisión (1ra Ed). Caracas, Venezuela: Fundación Sypal-Fundacite Anzoátegui.

Benedet, M. J. (2012). Neuropsicología Cognitiva. Aplicaciones a la clínica y a la investigación - 
Fundamento teórico y metodológico de la Neuropsicología Cognitiva (1ra Ed.). Madrid, España: Instituto de Migraciones y Servicios Sociales.

Blanes, A. (2016). La Teoría de las Inteligencias Múltiples (1ra Ed.). Barcelona, España: Bioinformática.

Carrillo, M. E., \& López, A. (2014). The multiple intelligence theory for the teaching of languages. Revista $\begin{array}{lllll}\text { de Educación: } & \text { Contextos } & \text { Educativos, } & \text { 17(1), }\end{array}$ https://doi.org/http://dx.doi.org/10.18172/con.2594

Castro, Y. M., Fonseca, L., \& Reyes, L. (2014). La didáctica como estrategia pedagogica para fortalecer el desarrollo de las inteligencias múltiples en los niños y niñas de la Institucion Educativa Rafael Uribe Uribe Sede Mampujan de Maria La Baja Bolivar. (Tesis de grado). Programa de Ciencias Sociales y Educación, Universidad del Tolima. Ibagué, Tolima, Colombia.

Chura, E. (2016). Psicología Humana (1ra Ed.). Arequipa, Perú: Chaparro Editores.

Contreras, K., \& Sotomayor, J. (2013). Psicología. Lima, Perú: Lumbreras Editores.

Cordero, G. (2007). Educación y Humanismo. Revista PRAXIS: Antropología, Cultura y Educación, 59(1), $35-50$.

Cordura, C. (2013). El Humanismo. Revista Chilena de Literatura, 84(1), 9-17.

Cuevas, R. (2011). Psicología Educativa (1ra Ed.). Lima, Perú: Editorial San Marcos.

Emst-Slavit, G. (2001). Education for All: Gardner's Multiple Intelligences Theory. Revista de Psicología de la PUCP, 19(2), 319-331.

Fernández, S., Paúl, N., \& Maestú, F. (2003). El papel de la Neuropsicología en la formación del Psicólogo. Revista de Psicología y Psicopedagogía de la Universidad Camilo José Cela, 67-80.

Gallegos, R. (1999). Educación Holística: Pedagogía del amor universal (1ra Ed). México: Editorial Pax México.

Gallegos, S. (2009). La teoría de las inteligencias múltiples en la enseñanza-aprendizaje de español como lengua extranjera. (Tesis de doctorado). Facultad de Filosofía, Universidad de Salamanca. Salamanca, España.

Gardner, H. (1983). Las Inteligencias Múltiples Estructura de la Mente (2da Ed.). Bogotá, Colombia: Fondo de Cultura Económica.

Gluyas, R. I., Esparza, R., \& Romero, M. (2015). Holistic educative model: a proposal for aiming to strengthen integral training of human being. Revista Actualidades Investigativas en Educación, 15(3), $1-25$.

González, D. J. (2003). ¿Qué es la inteligencia humana? Revista Cubana de Psicología, 10(1), 39-49.

Huerta, R., Giles, M., \& Rojas, P. (2010). Las inteligencias múltiples y el aprendizaje de las diversas áreas curriculares en los estudiantes del $4^{\circ}$ y $5^{\circ}$ ciclo de primaria del colegio experimental "Víctor Raúl Oyola Romero" de la Universidad Nacional de Educación. (Tesis doctoral). Dirección del Instituto de Investigación, Universidad Nacional de Educación Enrique Guzmán y Valle La Cantuta. Lima, Perú.

Llacsa, L. A. (2011). Relación entre inteligencias múltiples y elección vocacional en los estudiantes de la Institución Educativa Secundaria Gran Unidad Escolar San Carlos de la ciudad de Puno. (Tesis de pregrado). Universidad Nacional del Altiplano. Puno, Perú.

Macías, M. A. (2002). Las múltiples inteligencias. Psiclogía desde el Caribe Revista de la Universidad del Norte, 10(1), 27-38. 
Maldonado, J. J. (2014). Influencia de las inteligencias múltiples en las habilidades emprendedoras: estudio de casos de éxito en Extremadura. (Tesis de doctorado). Departamento de Psicología y Antropología de la Universidad de Extremadura. Badajoz, España.

Maquera, B. (2017). Las inteligencias múltiples y el rendimiento académico en estudiantes de la Institución Educativa Secundaria Emilio Romero Padilla Chucuito 2015. (Tesis de maestría). Universidad José Carlos Mariátegui. Moquegua, Perú.

Morris, C. G., \& Maisto, A. A. (2005). Introducción a la psicología (12ava Ed). Juárez, México: PEARSON Educación.

Papalia, D. E. (2009). Psicología (3ra Ed). DF, México: McGRAW-HILL Interamericana Editores.

Pintrich, P. R. (2000). Metas múltiples, vías múltiples: el papel de la orientación de la meta en el aprendizaje y el logro. Revista de Psicología Educativa, 92(3), 544-555.

Portellano, J. A. (2005). Introducción a la Neuropsicología (1 ra Ed.). Madrid, España: McGRAWHILL/Interamericana de España.

Romero, G. A. (2009). La pedagogía en la educación. Revista de Innovación y Experiencias Educativas, 19.

Rosas, M. E. (1996). Psicología (1 ra Ed). Lima, Perú: Editorial Brasa SA.

Sánchez, L. (2015). La teoría de las inteligencias múltiples en la educación. Sección de Investigaciones de la Universidad Mexicana, 1-14.

Shannon, A. M. (2013). La teoría de las inteligencias múltiples en la enseñanza de español. (Tesis de maestría). Universidad de Salamanca. Salamanca, España.

Stecconi, C. (2006). Inteligencias múltiples y el cuestionario de autoevaluación. Revista Calidad de Vida, l(5), 147-164.

Suárez, J., Maiz, F., \& Meza, M. (2010). Inteligencias múltiples: una innovación pedagógica para potenciar el proceso de enseñanza aprendizaje. Revista de Investigación y Posgrado de la Universidad Pedagógica Experimental Libertador, 25(1), 81-94.

Tirapu, J. (2011). Neuropsicología, Neurociencia y las Ciencias «PSI». Cuadernos de Neuropsicología, 1124.

Velasco, A. (2009). Humanismo (1 ra Ed.). Ciudad de México, México: Instituto de Investigaciones Sociales UNAM.

Villamizar, G., \& Donoso, R. (2013). Definiciones y teorías sobre inteligencia. Revisión histórica. Revista Psicogente, 16(30), 407-423.

Woolfolk, A. (2010). Psicología Educativa (3ra Ed.). DF, México: Pearson Educación. 